\title{
The Role of Lymphadenectomy in Minimally Invasive Urologic Oncology
}

\author{
Alon Z. Weizer, M.D., M.S., and Jeffrey S. Montgomery, M.D., MHSA
}

\begin{abstract}
Growing data suggest that lymphadenectomy is a critical component of the surgical management of urologic malignancies with both diagnostic and therapeutic benefit. The increased use of minimally invasive approaches for the management of urologic malignancies may be in conflict with the need for more extensive lymphadenectomy with these procedures. The purpose of this review is to summarize the current evidence for lymphadenectomy as a staging and/or therapeutic procedure for urologic oncology procedures, summarize the feasibility of lymphadenectomy for these disease processes using a minimally invasive approach, and make recommendations regarding management of these disease processes using minimally invasive approaches. A literature review using MESH terms to identify literature on lymphadenectomy in urologic oncology (including minimally invasive urologic oncology procedures) was performed. This literature was the summarized by disease process and by its relation to minimally invasive procedures. The literature demonstrates that lymphadenectomy can successfully duplicate that performed for open urologic oncology procedures. The application of minimally invasive approaches, however, requires advanced skills to be able to accomplish this.
\end{abstract}

\section{Introduction}

$\mathbf{L}$ YMPHADENECTOMY has long been held as a fundamental principle of surgical oncology. Because solid organ cancers typically spread in a stepwise fashion via the lymphatic system, adequate lymphadenectomy can be crucial to eradicating disease. By extension of this philosophy, there is a growing body of literature that documents the role of lymphadenectomy in the management of urologic malignancies.

There are several problems with this literature and the arguments for lymphadenectomy, however. First, too often the reported findings rely on retrospective series or administrative data cohorts. Unfortunately, when lymphadenectomy is put to the test in randomized controlled studies in other disease processes, the survival advantages do not bear out (eg, gastric, pancreas), so we must evaluate the current data with caution. ${ }^{1-3}$ Second, there continues to be no metric (ie, number of lymph nodes, lymph node density, specific template) to guide adequate lymph node dissections for many urologic malignancies. While specialty training in urologic oncology exists, most urologic cancers are managed by urologists without specialty training. ${ }^{4}$ The lack of data and defined metrics make lymphadenectomy resemble dogma instead of proven standard procedure for the surgical management of urologic malignancies.

To complicate matters, the increased use of minimally invasive techniques for the management of almost every urologic malignancy by a growing number of urologists has further strained the fundamental principles of lymphadenectomy in the surgical management of malignancies. Both the incorporation of laparoscopy into the majority of urology residency training programs 5 and the rapid adoption of robotassisted surgery have increased the use of minimally invasive approaches for urologic malignancies. The advantages advocated include shorter convalescence, decreased blood loss, and equivalent perioperative outcomes. Oncologic principles, however, are often compromised while refining the minimally invasive approach early in the adoption of the technique.

With these controversies in mind, this review will focus on the role of lymphadenectomy in minimally invasive urologic oncology. We will review the current level of evidence for lymphadenectomy for the most common urologic malignancies, defining its role as either therapeutic, staging, or both. We will review the feasibility of lymphadenectomy for minimally invasive urologic oncology procedures (laparoscopic or robot-assisted) and make recommendations for the application and extent of lymphadenectomy for minimally invasive urologic oncology procedures.

\section{Therapeutic Lymphadenectomy}

\section{Penile cancer}

Current evidence for lymphadenectomy. Penile cancer is a rare malignancy in the United States but has a much higher

Division of Urologic Oncology and Minimally Invasive Surgery, Department of Urology, University of Michigan, Ann Arbor, Michigan. 
incidence in parts of South America and Africa. ${ }^{6}$ A majority of penile cancers are squamous-cell carcinoma that are related to poor hygiene. While noninvasive disease is typically managed in an effort to preserve the organ and its function, invasive disease often necessitates partial or total penectomy. The depth of invasion locally combined with CT of the abdomen and pelvis and identification of palpable inguinal lymphadenopathy can assist in the accurate staging of the disease.

Many patients present initially with enlarged inguinal lymph nodes that are related to inflammation or secondary infection of the primary tumor. Traditionally, patients are placed on antibiotics for 4 to 6 weeks after management of the primary disease with reassessment of the inguinal adenopathy, assuming that the lymphadenopathy is inflammatory in nature; however, multiple series suggest that even patients with lower grade and stage disease may harbor micrometastases. ${ }^{6}$

Currently, patients with $\geq \mathrm{pT}_{2}$ or $\mathrm{T}_{1}$ disease with adverse histologic features should be considered for bilateral inguinal lymph node dissection (ILND) ${ }^{6}$ (see also Guidelines on Penile Cancer, www.uroweb.org). There is a clear survival advantage to lymphadenectomy in this setting $v s$ waiting for development of palpable disease. ${ }^{7}$ In the circumstance of positive nodes that are identified during the superficial ILND, the deep inguinal nodes should be removed in the same setting. Removal of micrometastatic disease may be curative and will certainly help to manage the local disease that, if left untreated, may have dire consequences. ${ }^{8}$

In the setting of palpable disease or enlarged lymph nodes seen on CT, there is an imperative to perform a lymph node dissection. Inguinal lymphadenectomy should still be performed even if inguinal adenopathy resolves after antibiotic therapy, based on the stage of the initial tumor. ${ }^{6}$ Most surgeons use a modified ILND as described by Catalona. ${ }^{9}$ The modified technique is designed to reduce the morbidity that is associated with the standard ILND by preserving the saphenous vein during a superficial dissection, reducing the boundaries of dissection to decrease the risk of skin flap necrosis and decreasing the incisional length.

Results have demonstrated that patients who are most likely to be cured by ILND are those with nonpalpable inguinal lymph nodes that contain micrometastatic disease. The probability of cure decreases from $100 \%$ to $73 \%$ at 3 years when positive nodes are palpable before surgery. ${ }^{8}$ When enlarged pelvic lymph nodes are identified on $\mathrm{CT}$, the management is somewhat more controversial. While some series advocate proceeding with pelvic lymphadenectomy before
ILND, the probability of cure in patients with pelvic lymph node metastases is exceedingly low. In patients with borderline pelvic lymphadenopathy, however, a limited pelvic lymph node dissection (PLND) or percutaneous biopsy can aid in the decision process. If the pelvic lymph nodes are negative, an inguinal lymphadenectomy can still be curative. If the pelvic lymph nodes are positive, however, then inguinal lymphadenectomy is primarily palliative with adjuvant radiation and/or chemotherapy considered for selected patients. Protzel and colleagues ${ }^{6}$ recommend completing a PLND when two or more positive nodes or extranodal extension of disease is identified on the ILND, but this practice is not widespread.

Feasibility of minimally invasive approaches. ILND is a morbid procedure. Even with a modified template, the risk of a complication can be as high as $50 \%{ }^{10}$ and can include wound infection, flap necrosis, lymphocele, lymphedema, thrombophlebitis, and hemorrhage. In an effort to reduce the morbidity of this procedure, Bishoff and associates ${ }^{11}$ described a laparoscopic technique for superficial ILND. Several other series have been reported on the use of video endoscopic inguinal lymphadenectomy (VEIL) procedures. These series are summarized in Table 1. Of note, Tobias-Machado and coworkers ${ }^{12}$ failed to identify a difference in the number of lymph nodes obtained via an open or endoscopic approach.

Minimally invasive approaches to the management of pelvic lymphadenopathy for penile cancer were first described in 1994. At that time, this group performed three laparoscopic pelvic lymphadenectomies for stage $T_{3}$ disease and removed a mean of eight lymph nodes without complications. ${ }^{13}$ A large body of literature, mostly related to prostate cancer, has demonstrated the efficacy and feasibility of laparoscopic PLND. Its use in the management of penile cancer remains controversial.

Recommendations. ILND in penile cancer can be curative. In patients with nonpalpable disease, a laparoscopic approach can be considered by surgeons with experience with inguinal anatomy and laparoscopic techniques. In the small reported series, it appears to reduce the morbidity of the operation. Consideration should be given to cadaveric dissection before using this approach in patients, as recommended by Bishoff. Frozen section pathologic evaluation should be obtained of the superficial nodes and a deep ILND should be performed if nodal metastasis is identified. This can be accomplished laparoscopically, depending on the surgeon's comfort level. For palpable disease, Bishoff has raised concern

Table 1. Results of Minimally Invasive Inguinal Lymphadenectomy for Penile Cancer

\begin{tabular}{|c|c|c|c|c|c|}
\hline Reference & $N$ (groins) & No. lymph nodes & No. + nodes & Complications & Follow-up \\
\hline $\begin{array}{l}\text { Sotelo }^{14} \\
\text { Tobias-Machado }^{12}\end{array}$ & $\begin{array}{c}14 \\
10 \text { open } \\
20 \text { laparoscopic }\end{array}$ & $\begin{array}{r}9(4-15) \\
9.7(6-14) \\
10.8(7-16)\end{array}$ & $\begin{array}{c}\text { Not recorded } \\
14\end{array}$ & $\begin{array}{l}\quad \text { Lymphocele } 23 \% \\
\text { Laparoscopic vs open: } \\
\text { Lymphocele } 10 \% \text { vs } 20 \% \\
\text { Skin events: } 5 \% \text { vs } 50 \% \\
\text { Hematoma } 5 \% \text { vs } 0 \%\end{array}$ & $\begin{array}{l}\text { No local or systemic } \\
\text { recurrence at mean } \\
32 \text { months }\end{array}$ \\
\hline $\begin{array}{l}\text { Josephson }^{73} \\
\text { Weizer and } \\
\text { Montgomery }\end{array}$ & $\begin{array}{c}2 \text { robot-assisted } \\
5\end{array}$ & $\begin{array}{c}9.5(9-10) \\
7.8\end{array}$ & $\begin{array}{l}0 \\
1\end{array}$ & $\begin{array}{l}\text { None } \\
\text { Lymphocele } 20 \% \\
\text { Hemorrhage } 20 \%\end{array}$ & $\begin{array}{l}\text { Not recorded } \\
\text { Not mature }\end{array}$ \\
\hline
\end{tabular}


for a higher risk of complications because of limited experience. For this patient population, a laparoscopic technique should be used only by the most experienced surgeons. ${ }^{14}$

The decision of whether and when to use PLND remains controversial. Experience with laparoscopic and robotassisted PLND for prostate and bladder cancer makes a minimally invasive approach attractive if the surgeon deems pelvic lymphadenectomy appropriate. In this setting, it is likely to reduce the morbidity associated with this procedure.

\section{Testis cancer}

Current evidence for lymphadenectomy. Retroperitoneal lymph node dissection (RPLND) continues to play a critical role in the management of testicular cancer, especially in patients with nonseminomatous germ-cell tumors. A critical disconnect is that most urologic oncologists who are performing this as an open procedure view RPLND as a therapeutic intervention, while many performing laparoscopic RPLND view it as a staging procedure, with adjuvant chemotherapy ultimately having a role in controlling the disease. While a detailed review comparing surveillance, chemotherapy, and RPLND for low-risk disease and the use of RPLND after chemotherapy for high-risk disease is beyond the scope of this review, the 2009 National Comprehensive Cancer Network (NCCN) guidelines serve as an excellent resource for the management of the disease based on literature review and expert opinion (NCCN.org). Here, the literature on minimally invasive RPLND will be reviewed and contrasted to known benchmarks for standard management of the disease.

Feasibility of minimally invasive approaches. Clinical stage I/II. Open RPLND is considered an effective therapeutic option for the management of stage IA or B, IIA or IIB. For stage IA disease, surveillance should be considered, because retroperitoneal recurrence will not develop in $70 \%$ to $80 \%$ of patients and the remainder can undergo salvage therapy with chemotherapy or radiation therapy without detriment to overall survival. Long-term evaluation of surveillance is needed, however, because many physicians do not adhere to established surveillance guidelines. ${ }^{15}$ For patients with stage
IB disease, two cycles of adjuvant bleomycin, etoposide, and platinum (BEP) chemotherapy can be considered in addition to surveillance or RPLND (NCCN.org). For patients with stage IIA or IIB disease with elevated tumor markers, three cycles of BEP or four cycles of etoposide and cisplatin chemotherapy are indicated. If tumor markers are normal, upfront RPLND is another alternative (NCCN.org).

The extent of RPLND as a primary therapeutic modality depends on the stage. For patients with stage IA or IB disease, most surgeons advocate for dissection according to establish templates. ${ }^{16}$ In the setting of enlarged retroperitoneal nodes, however, a bilateral template is recommended by most urologic oncologists with a nerve-sparing approach preferred as long as it does not compromise disease control (NCCN.org). For patients with stage IIA or IIB disease, an open, bilateral, nerve-sparing RPLND is recommended by the NCCN guidelines (NCCN.org).

With this context in mind, Table 2 summarizes the results of patients who are undergoing laparoscopic RPLND for marker negative stage I and II disease. There is variability in how each series approaches RPLND for stage I, IIA, and IIB disease. Most use a modified template with no adjustment made if positive nodes are identified at the time of the procedure. In addition, most series do not advocate for the resection of the tissue dorsal to the great vessels citing, mapping studies performed by Albqami and Janetschek ${ }^{17}$ that demonstrate that patients with positive nodes dorsal to the great vessels tend to have positive nodes ventral to the great vessels. Finally, most series use adjuvant chemotherapy in the setting of node-positive disease.

While recurrence-free survival and overall survival seem comparable to patients who are undergoing open RPLND, the primary difference is the use of postoperative chemotherapy for patients with pathologic stage II disease. While this does not appear to compromise outcomes, the argument of surgeons performing open surgery is that RPLND should be performed with the goal of avoiding chemotherapy and its long-term sequelae. ${ }^{18}$ Rassweiler and coworkers ${ }^{19}$ recently review 34 published series of open vs laparoscopic RPLND. There was no significant difference detected between laparoscopic $(n=557)$ and open $(n=761)$ approaches in terms of

Table 2. Results of Laparoscopic Retroperitoneal Lymph Node Dissection for Stage I and II Disease

\begin{tabular}{|c|c|c|c|c|c|c|c|c|c|}
\hline Series & $N$ & Stage & Template & $\begin{array}{l}\text { Retrovascular } \\
\text { dissection }\end{array}$ & $\begin{array}{l}N \text { positive } \\
\text { nodes }\end{array}$ & $\begin{array}{l}\text { Chemo for } \\
\text { node }+\end{array}$ & $\begin{array}{c}\text { Mean lymph } \\
\text { nodes }\end{array}$ & $\begin{array}{l}\text { Follow-up } \\
\text { (mos) }\end{array}$ & Results \\
\hline Bhayani $^{74}$ & 29 & I & Unilateral & No & 12 & $10 / 12$ & 20 & 69.6 & $\begin{array}{l}\mathrm{N}_{0}: 2 \text { recurrences } \\
\mathrm{N}+: 1 \text { recurrence }\end{array}$ \\
\hline Castillo $^{75}$ & 111 & I & & & 21 & $21 / 21$ & & 34 & $4.5 \%$ \\
\hline Albqami $^{17}$ & 103 & I & Unilateral & No & 26 & $26 / 26$ & NR & 62 & $\begin{array}{l}\mathrm{N}_{0}: 6 \text { recurrence } \\
\mathrm{N}+: \text { none }\end{array}$ \\
\hline Abdel-Aziz ${ }^{76}$ & 22 & I & Unilateral & No & 7 & $5 / 7$ & 17 & 12 & $\begin{array}{l}1 \text { recurrence } \\
\text { (outside template) }\end{array}$ \\
\hline Neyer $^{77}$ & 136 & I & Unilateral & NR & 25 & $25 / 25$ & NR & NR & $5.9 \%$ \\
\hline Nielsen $^{78}$ & 120 & $\mathrm{I}$ & Unilateral & Yes & 46 & $36 / 46$ & 20 & 29 & $\begin{array}{l}\mathrm{N}_{0}: 2 \text { recurrence } \\
\mathrm{N}+\text { : none }\end{array}$ \\
\hline Cresswell $^{79}$ & 79 & I & Unilateral & No & 19 & $19 / 19$ & 14 & 84 & $9 \%$ recurrence \\
\hline Skolarus ${ }^{80}$ & 26 & I/IIA,B & Unilateral $^{\mathrm{a}}$ & Yes & 6 & $5 / 6$ & 23.8 & 23.7 & None \\
\hline Steiner $^{81}$ & 23 & I/IIA,B & Bilateral $^{\mathrm{b}}$ & Yes & 6 & 0 & 22 & 17.2 & $\mathrm{~N}_{0}: 1$ recurrence \\
\hline
\end{tabular}

a If positive node identified intraoperatively, a bilateral procedure is performed.

'Patients before 1992, open bilateral template.

Chemo = chemotherapy; NR = not recorded . 
Table 3. Postchemotherapy Retroperitoneal Lymph Node Dissection

\begin{tabular}{lccclccc}
\hline Series & $N$ & Stage & Template & Complications & Teratoma & Follow-up (mos) & Recurrence \\
\hline Steiner $^{82}$ & 68 & IIA,B,C & Unilateral & None & $38 \%$ & 98.6 & 1 \\
Steiner $^{83}$ & 59 & IIA & Unilateral & NR & $39 \%$ & 1 \\
Calestroupat $^{84}$ & 26 & IIA,B,C & Unilateral & 9 (8 lymphovascular) & $19 \%$ & 27 & 0 \\
Steiner $^{81}$ & 19 & IIB & Bilateral & Lymphocele & $21 \%$ & $31.3 \%$ & 32.7 \\
Permpongkosol $^{85}$ & 16 & II & Unilateral & $7(43.8 \%)$ & $37.5 \%$ & 26 & 1 \\
Maldonado-Valadez $^{86}$ & 16 & II & Unilateral & 1 (pneumonia) & & 2 \\
\hline
\end{tabular}

$\mathrm{NR}=$ not recorded

retroperitoneal relapse $(1.3 \%$ vs $1.4 \%)$, in field relapse $(0 \%$ vs $0.45 \%$ ), distant progression ( $3.3 \%$ vs $6.1 \%$ ), and biochemical failure $(0.9 \%$ vs $1.1 \%)$; however, a higher percentage of nodepositive disease was identified with open RPLND. Of interest, the authors reported equal rates of adjuvant chemotherapy $(29 \%$ vs $31 \%)$ in both cohorts. ${ }^{19}$

It may seem that for stage I and II disease, the results are comparable; however, several issues remain. First, the above findings are from high-volume centers, and there is clearly an issue of a learning curve that could compromise a clinician's early results. ${ }^{19}$ Second, the technique and the approach to address intraoperative findings have not been standardized. To garner widespread acceptance as a viable surgical option, it is crucial to prove that laparoscopic RPLND replicates the open technique in terms of extent of dissection and use of a bilateral template when dictated by intraoperative findings. Suboptimal dissection threatens young men with unnecessary exposure to chemotherapy and its long-term sequelae as well as the risk of residual retroperitoneal teratoma. ${ }^{18,20}$ The cardiovascular and secondary malignancy risks are not trivial in patients who are receiving platinum-based chemotherapy, and while there are trends in Europe toward single courses of chemotherapy to manage stage I disease, the long-term results are not available. In addition, the presence of unresected teratoma after RPLND and chemotherapy and need for reoperation are associated with higher complication rates.

Postchemotherapy management. Table 3 summarizes the results of postchemotherapy laparoscopic RPLND in recent series. Most series are small. Steiner and associates has reported several series during different periods and, to the best of our knowledge, these studies represent mutually exclusive patients. Most dissections in this setting involve resection of the residual mass and an ipsilateral template. While risk of recurrence appears minimal with relatively short follow-up, the high percentage of patients with residual tumor and mature teratoma at the time of postchemotherapy RPLND raises concerns for inadequate initial resection. The current NCCN guidelines recommend a bilateral nerve-sparing RPLND in the setting of the presence of residual mass after chemotherapy based on level IIB evidence (NCCN.org).

These series from surgeons with extensive laparoscopic RPLND experience, however, demonstrate that this approach is feasible with similar complication rates as described in open postchemotherapy RPLND.

Recommendations. While there is no level I evidence to support this practice, patients with stage I nonseminomatous germ-cell tumors can be treated with a template-based laparoscopic RPLND. To replicate the open technique, however, laparoscopic surgeons should be committed to performing bilateral template surgery when indicated, dissecting posterior to the vessels and counseling patients regarding the risks and benefits of adjuvant chemotherapy for pathologic stage IIA disease. It is the onus of the surgeon to determine whether the dissection was adequate and if chemotherapy is indicated. Only with these actions will laparoscopic surgeons move toward equivalence with open RPLND.

A randomized controlled trial that compares open and laparoscopic RPLND is likely not feasible. In addition, most series echo that extensive laparoscopic experience is essential to the successful performance of laparoscopic RPLND. The use of robotic techniques may further aid surgeons in a more careful dissection that includes nerve preservation and dissection posterior to the vessels with ligation of the lumbar vessels, although only limited reports are available. ${ }^{21}$

\section{Staging/Likely Therapeutic}

\section{Bladder cancer}

Current evidence for lymphadenectomy. There is evidence that PLND in bladder cancer is both diagnostic and therapeutic, but there is still controversy over the extent of lymph node dissection needed at the time of cystectomy. Some surgeons believe that lymphadenectomy is only a diagnostic procedure, and an extended lymph node dissection unnecessarily prolongs an already significant surgery. These surgeons tend to remove a sampling of nodal tissue, but it has been shown that currently up to $16 \%$ of patients undergoing cystectomy get no node dissection at all. ${ }^{22}$

Contrary to this, there are those surgeons who think that lymphadenectomy during cystectomy not only provides valuable disease staging information, but it is also a therapeutic procedure with patient survival implications. These surgeons tend to perform extended lymph node dissections, clearing the nodal tissue from the internal, external, and common iliac, obturator, presacral, para-aortic and paracaval regions.

If there is a survival advantage to an extended lymph node dissection, it is likely because of improved local and regional cancer control, removing all nodes with gross and micrometastatic disease and more accurately identifying those patients who may benefit from adjuvant chemotherapy. Leissner and associates ${ }^{23}$ performed a prospective evaluation of lymph node metastases in 290 patients with bladder cancer who underwent cystectomy and an extended lymphadenectomy. They performed a rigorous extended PLND up to the inferior mesenteric artery and included the presacral lymph node packet as well. With this, their average node count was $43.1 \pm 16.1$. They identified nodal metastases in each of the 12 nodal regions they defined and also confirmed 
Table 4. Pelvic Lymph Node Dissection with Cystectomy: Results According to Surgical Approach

\begin{tabular}{|c|c|c|c|c|c|}
\hline Series & Approach & $N$ & Extent of dissection & Mean node count (range) & PLND operative Time \\
\hline Leissner $^{23}$ & Open & 290 & Extended & $43.1(11-99)$ & NR \\
\hline \multirow[t]{2}{*}{ Stephenson $^{87}$} & Open & 50 & Limited & 16 & NR \\
\hline & Laparoscopic & 50 & & 15 & \\
\hline \multirow[t]{2}{*}{ Finelli $^{31}$} & Laparoscopic & 22 & Extended & $18(6-30)$ & $90 \mathrm{~min}$ \\
\hline & & & Limited & $6(1-15)$ & $45 \mathrm{~min}$ \\
\hline \multirow[t]{2}{*}{ Abraham $^{33}$} & Laparoscopic & 16 & Extended & $16.5(7-26)$ & NR \\
\hline & Robot-assisted & 10 & & $22.3(13-42)$ & \\
\hline \multirow[t]{2}{*}{ Wang $^{35}$} & Open & 21 & Limited & $20(8-38)$ & NR \\
\hline & Robot-assisted & 33 & & $17(6-32)$ & \\
\hline Guru $^{88}$ & Robot-assisted & 20 & $\begin{array}{l}\text { Extended } \\
\text { Limited }\end{array}$ & $13(6-26)$ & $44 \mathrm{~min}$ \\
\hline Pruthi $^{34}$ & Robot-assisted & 50 & $\begin{array}{l}\text { Extended } \\
\text { Limited }\end{array}$ & $19(8-37)$ & NR \\
\hline Woods ${ }^{89}$ & Robot-assisted & 27 & Extended & $12.3(7-20)$ & NR \\
\hline
\end{tabular}

$\mathrm{PLND}=$ pelvic lymph node dissection; $\mathrm{NR}=$ not recorded.

that bladder cancer metastasizes in a bilateral fashion even in patients with unilateral disease.

Stein and colleagues ${ }^{24}$ revealed that the lymph node count and density of positive lymph nodes removed during lymphadenectomy for bladder cancer had survival implications. Patients with $\mathrm{pN}+$ disease with 15 or fewer nodes removed had a $25 \%$ 10-year recurrence-free survival compared with $36 \%$ when more than 15 nodes were removed. They defined the concept of node density in bladder cancer, showing that patients with a $\mathrm{pN}+$ node density $<20 \%$ had a $43 \% 10$-year recurrence-free survival compared with $17 \%$ for those with a density $>20 \%$. Several other authors have shown that the total number of lymph nodes involved with metastatic disease and the completeness of dissection are important factors in patient survival after cystectomy. ${ }^{25-29}$

Feasibility of minimally invasive approaches. The laparoscopic approach for cystectomy is technically challenging and an option only for the most skilled laparoscopic surgeons. With robot-assisted surgery facilitating the uptake of a minimally invasive approach by a greater number of surgeons, intracorporeal cystectomy and lymphadenectomy has expanded greatly since the technique was initially described. ${ }^{30}$ Table 4 summarizes the primary series of laparoscopic and robot-assisted radical cystectomy in terms of lymph node yield. These data suggest that comparable lymph node dissections can be performed using minimally invasive approaches compared with open radical cystectomy and pelvic lymphadenectomy.

Extended PLND is achievable using both laparoscopic and robot-assisted approaches to radical cystectomy. Finelli and coworkers $^{31}$ reported on 11 patients who underwent a laparoscopic extended PLND after cystectomy with a median node count of 21 . This extended node dissection necessitated an additional hour of operative time compared with the limited dissection. Haber and $\mathrm{Gill}^{32}$ reported on 26 patients who underwent an extended laparoscopic PLND after radical cystectomy with median number of excised nodes of 21 (range 11-24). Abraham and coworkers ${ }^{33}$ completed a prospective comparison of 20 laparoscopic and 14 robot-assisted radical cystectomy patients with open ileal conduit urinary diversions. There were no significant differences in terms of mean operative time, but there was less blood loss, fewer transfusions, fewer postoperative complications, and an earlier return to bowel function in the robotic group. There was no significant difference in the number of lymph nodes removed in the two groups, with a mean of 22.3 nodes in the robotic group and 16.5 in the laparoscopic group in those patients in which an extended PLND was performed.

With the dissemination of robotic techniques, surgeons with varying degrees of laparoscopic experience are performing robot-assisted cystectomies. During the initial cases, inexperience and unfamiliarity with the approach can lead to significantly longer operative times. There is concern that the extent of lymph node dissection is sacrificed or lymphadenectomy is not performed at all to avoid prolonging the case. Pruthi and Wallen, ${ }^{34}$ however, reported on their learning curve for robotassisted cystectomy and found that their lymph node yield remained stable throughout their first 50 patients with an average of 19 nodes retrieved. Wang and colleagues ${ }^{35}$ performed a prospective comparison of 54 consecutive patients who underwent either open radical cystectomy $(n=21)$ or robotassisted cystectomy $(n=33)$. They found that the median number of lymph nodes removed was similar in the two approaches (20 vs 17, favoring the open cohort).

Recommendations. Although much emphasis is placed on node counts in radical cystectomy as a surrogate of completeness of lymphadenectomy, several variables influence the number of lymph nodes found in the final pathologic specimen. Individual patient anatomic variation, the commitment of the pathologist to identify nodes, and submission of nodes en bloc or in individual packets can all affect the total number of lymph nodes in the final pathologic report. The extent and thoroughness of node dissection, not just total node count, are important variables to consider. Although not yet confirmed in a randomized controlled trial, there are substantial data that indicate a survival advantage associated with an extended PLND compared with a limited PLND.

The goal of cystectomy with PLND, whether approached open, laparoscopically or robotically, should be thoroughness of technique to assure complete removal of the bladder and nodes, preventing positive surgical margins and local recurrences. Several reports have shown that the laparoscopic 
and robot-assisted approaches afford the surgeon the ability to complete an extended PLND, including the external iliac, internal iliac, common iliac, obturator, para-aortic, paracaval and presacral node packets. Although long-term data are still needed, it seems that these techniques offer the opportunity to provide the advantages of minimally invasive surgery to bladder cancer patients without sacrificing oncologic principles, as long as surgeons using these approaches are persistent and fastidious in their technique.

\section{Prostate cancer}

Current evidence for lymphadenectomy. A prostate cancer patient's risk for lymph node metastasis is predicted by the clinical stage of the prostate cancer, the preoperative prostate-specific antigen (PSA) level, and the biopsy Gleason score. ${ }^{36}$ Although it is commonly thought that the obturator nodes are the primary sites for nodal metastases in prostate cancer, several anatomic and lymphography studies have established that the prostate drains to four main node groups: The internal iliac group as the primary, the obturator nodes as the secondary, the external iliac as the tertiary, and the presacral nodes as the quarternary landing sites. ${ }^{37-39}$ Bader and associates ${ }^{40}$ reported on 365 patients with clinically localized prostate cancer who underwent prostatectomy and lymphadenectomy that included the tissue over the external iliac vein, the internal iliac vessels, and in the obturator fossa. The median number of nodes removed was 21 (range 6-50). Nodepositive disease was identified in $24 \%$ of patients, with $58 \%$ of these patients having positive nodes within the internal iliac packet with isolated metastatic spread in $20 \%$. An obturator lymph node dissection alone would have missed metastatic nodes in $39 \%$ of patients. Heidenreich and colleagues ${ }^{41}$ compared 103 patients who underwent an extended PLND with 100 patients who underwent the standard lymphadenectomy (ie, external iliac and obturator lymph nodes). Nodal metastases were found in $26.6 \%$ of the extended group and only $12 \%$ of the standard node dissection group. Positive nodes were outside standard regions of dissection in $42 \%$ of patients. A standard lymph node dissection understaged a significant percentage of prostate cancer patients.

There is a growing body of evidence that indicates that an extended PLND during prostatectomy is associated with a survival advantage. Bader and coworkers ${ }^{40}$ showed that with their extended PLND, of the 367 patients with clinically organ-confined prostate cancer, $25 \%$ had lymph node metastases. At a median follow-up of 45 months and without adjuvant therapy, $39 \%$ of patients with one node positive remained without signs of clinical or biochemical progression vs $12 \%$ for those patients with two or more positive nodes. The authors concluded that their extended PLND is not only a more accurate staging procedure but also may have a positive impact on disease progression and patient survival.

Reviewing the outcomes of more than 13,000 men who underwent prostatectomy as primary therapy logged in the Surveillance, Epidemiology and End Results (SEER) database from 1988 to 1991, Joslyn and Konety ${ }^{42}$ found that those men who had at least four nodes removed at the time of prostatectomy had a lower rate of prostate cancer-specific death at 10 years than those who did not undergo lymphadenectomy. A more extensive dissection $(10+$ nodes removed) was associated with a significantly lower risk of prostate-cancer death even when the analysis was restricted to those patients who were node negative. Even with pathologic node-negative disease, a more extended PLND may remove micro-metastatic disease, improving disease free survival. ${ }^{43}$

Schumacher and colleagues ${ }^{44}$ reviewed 122 node-positive patients who were thought to have organ-confined disease by preoperative staging studies. A median of 22 nodes were removed, median PSA was $16 \mathrm{ng} / \mathrm{mL}, 76 \%$ were $\mathrm{pT}_{3}-\mathrm{pT}_{4}$, and $50 \%$ had seminal vesicle invasion. With the extended lymph node dissection, median 5- and 10-year survival was $84.5 \%$ and $60.1 \%$, respectively, and patients with $\leq 2$ nodes positive fared better.

Lymph node dissection at the time of prostatectomy may be more crucial for those patients with intermediate- or highrisk disease, and if a node dissection is performed, an extended dissection should be preferred.

Contrary to this, DiMarco and coworkers ${ }^{45}$ found that the number of lymph nodes obtained at lymphadenectomy was not associated with improved rates of PSA progression, systemic progression, or disease-specific survival after prostatectomy. Using the CaPSURE database, Berglund and associates ${ }^{46}$ found that a limited PLND at the time of prostatectomy did not significantly affect the rate of biochemical failure in low-, intermediate-, and high-risk groups. Controversy still exists over the proper extent of PLND at the time of prostatectomy.

Feasibility of minimally invasive approaches. One of the major criticisms of minimally invasive prostatectomy is that it may compromise the strict oncologic standards established with the open procedure. Although there are still no longterm data, several studies have shown that in regard to positive margin status and PSA recurrence, there is no significant difference between minimally invasive prostatectomy and open prostatectomy. ${ }^{47,48}$ Specific to lymphadenectomy, there is concern that minimally invasive surgeons may forgo or perform an incomplete PLND in the interest of shortening operative times, especially if the surgeon is inexperienced or infrequently performs the surgery. One study that evaluated a contemporary cohort of Medicare patients found that only $17 \%$ of men who were undergoing minimally invasive radical prostatectomy underwent a PLND compared with $83 \%$ for the open procedure, and there was significant surgeon and regional variability in performance of a PLND. ${ }^{49}$ Surgeons (ie $>40$ prostatectomies) in high-volume centers and surgeons in the western United States were more likely to perform PLND at the time of prostatectomy. This study raises serious concerns regarding the oncologic integrity of minimally invasive prostatectomy.

Several surgeons have documented that it is possible to complete a thorough laparoscopic or robot-assisted lymph node dissection at the time of prostatectomy. Zorn and colleagues $^{50}$ found that their mean nodal yield during robotassisted prostatectomy was 12.5 , which was comparable to their mean nodal yield on open prostatectomy of 15. Comparing their robot-assisted procedures with and without PLND, operative time, estimated blood loss, length of hospital stay, and complication rates were not significantly different between the two groups. They concluded that robot-assisted PLND can be performed safely and efficiently, with results similar to those for open PLND.

Wyler and associates ${ }^{51}$ performed a laparoscopic extended lymph node dissection with 123 consecutive prostatectomies 
and had a mean lymph node yield of 21 (range 9-55). Although a thorough extended PLND can prolong the operative time upward of 45 to 60 minutes, ${ }^{52}$ it can be performed safely with minimally invasive prostatectomy with limited added patient morbidity.

Recommendations. In addition to having a diagnostic benefit, patients who undergo a PLND with laparoscopic or robot-assisted prostatectomy may have improved disease recurrence and survival rates. An extended PLND, including the external iliac, obturator, and internal iliac nodes, reflects the true lymphatic drainage of the prostate, increases nodal yield, results in more accurate disease staging, and promotes early initiation of adjuvant therapy that has proven survival benefit. ${ }^{53}$ Surgeons may choose to forgo node dissection entirely in those patients with low-risk disease (ie, $\leq$ Gleason 6 disease, PSA $\leq 10 \mathrm{ng} / \mathrm{mL}$, clinical stage $\leq \mathrm{T}_{2 \mathrm{a}}$ ), because several studies have shown a low rate of node-positive disease in these persons. ${ }^{36,54,55}$ If lymphadenectomy is performed, however, a bilateral dissection is indicated even if a patient has disease isolated to one lobe of the prostate on biopsy. Prostate cancer is too frequently bilateral and has variable lymphatic drainage to safely perform unilateral node dissections. Ultimately, it is incumbent on those performing minimally invasive surgery to adhere to strict oncologic principles, maintain consistent PLND selection criteria, and perform a node dissection that most benefits the patient.

\section{Upper tract urothelial carcinoma (UTUC)}

Current evidence for lymphadenectomy. The use of lymphadenectomy in UTUC is mostly extrapolated from bladder cancer in which there is a clear staging and possible therapeutic benefit to those patients with micrometastatic disease (see above). The lymphatic drainage and therefore the probable sites of nodal spread, however, depend on the location of the tumor. Currently, there is no standardization to the approach and application of lymphadenectomy for UTUC.

Recent evidence has been obtained from an international, retrospective cohort study of patients with UTUC who were treated with nephroureterectomy. Roscigno and colleagues ${ }^{56}$ evaluated 1130 patients with presumed node-negative disease. Of these patients, 412 patients had $\mathrm{pN}_{0}$ disease, 578 (51.1\%) patients had $\mathrm{pNx}$ disease, and $140(12.4 \%)$ patients had node-positive disease. Except for those patients with $\mathrm{pT}_{1}$ disease, patients with $\mathrm{pN}_{\mathrm{x}}$ disease had worse 5-year diseasespecific survival than those with $\mathrm{pN}_{0}$ disease. Patients with $\mathrm{pN}+$ disease, however, had a worse 5-year disease-specific survival (33\% vs $58 \%$ for $\mathrm{pN}_{\mathrm{x}}$ disease and $70 \%$ for $\mathrm{pN}_{0}$ disease, $P=0.017)$. This study suggests that lymphadenectomy in UTUC has a staging role that could aid in the selection of patients for adjuvant chemotherapy. ${ }^{56}$

In a subset analysis of the same cohort, Roscigno and associates $^{57}$ evaluated 552 patients with clinically localized UTUC who underwent nephroureterectomy and lymphadenectomy. The number of lymph nodes removed was not associated with disease-specific survival in univariate or multivariable analysis. In patients with $\mathrm{pN}_{0}$ disease $(\mathrm{n}=412)$, however, the number of lymph nodes removed was an independent predictor of disease-specific survival, especially in patients with eight or more or more lymph nodes removed. While this would suggest that patients with lymph node positive disease already have systemic disease and that lymphadenectomy is purely a staging procedure, it also suggests that lymphadenectomy may be therapeutic in a subset of patients with localized disease and nodal micrometastases.

This same group of patients was used to define the concept of lymph node density in UTUC. Of 432 patients undergoing lymphadenectomy and nephroureterectomy, 135 (31\%) patients had lymph node metastases. Patients with a lymph node density of $\geq 30 \%$ were at greatest risk for disease recurrence (hazard ratio [HR] 1.8, $P=0.021$ ) and death (HR 1.7, $P=0.032$ ) after adjusting for grade and stage. This would suggest that in high-risk disease, positive nodes and lymph node density can be used to select patients who are appropriate for adjuvant chemotherapy. ${ }^{58}$

These data have been contradicted by Kondo and colleagues. ${ }^{59}$ They reviewed their experience with limited vs extended lymphadenectomy with nephroureterectomy based on mapping studies that were performed previously $(n=169)$. Overall, the patients who were undergoing resection of all primary sites of lymph node metastases did no better than the patients who were undergoing a limited lymph node dissection. When patients with $\geq \mathrm{pT}_{3}$ disease were evaluated, however, extensive lymphadenectomy was an independent predictor of disease-specific survival on multivariate analysis after adjusting for grade and stage (HR $0.29,95 \%$ confidence interval $0.11-0.73$ ). This study failed to establish a significant association between total node yield and patient survival, likely because of the limited number of patients with $\geq \mathrm{pT}_{3}$ disease.

The variation in results of the UTUC collaborative and the small series from Kondo and coworkers ${ }^{59}$ is likely a difference in lymphadenectomy technique. The former represents a cohort with multiple surgeons, dissection techniques, approaches, and likely extent of lymphadenectomy when performed. In this setting, patients with low-risk disease may not routinely receive lymphadenectomy while those with regionally advanced disease may more consistently undergo lymphadenectomy for staging purposes. In the latter study, deposits of lymph node metastases were previously mapped, ${ }^{60}$ encouraging the surgeons to be more vigilant and thorough with their lymphadenectomy in hopes of salvaging patients from metastatic disease. Similar to other urologic cancers, the true value of lymphadenectomy may be based on standardization of its use rather than lymph node totals.

Feasibility of minimally invasive approaches. In a separate analysis of the Upper Tract Urothelial Cancer Collaborative group, Capitanio and associates ${ }^{61}$ compared short-term oncologic results of patients who were undergoing open $(\mathrm{n}=979)$ vs laparoscopic $(\mathrm{n}=270)$ nephroureterectomy. After adjusting for disease stage, grade, and adverse histologic features, there was no statistically significant difference in overall and disease-specific survival between surgical approaches. Of note, $42.4 \%$ of patients who were undergoing open nephroureterectomy underwent lymphadenectomy vs $24.4 \%$ of the laparoscopic group. While the intent of this study was not to evaluate the impact of lymphadenectomy on survival, it is clear that in a nonstandardized cohort of patients, lymphadenectomy is less frequently performed in the laparoscopic than the open setting. The lack of identifiable difference in oncologic control between open and laparoscopic nephroureterectomy is echoed in multiple other studies. ${ }^{62-65}$ 
Despite the fewer number of patients undergoing lymphadenectomy with UTUC via a laparoscopic approach, the survival appears to be similar, after adjustment for disease severity, suggesting that these series all have a component of selection bias that is dampening the impact of lymphadenectomy. Busby and colleagues ${ }^{66}$ have demonstrated that an adequate lymphadenectomy can be performed during laparoscopic nephroureterectomy compared with the open surgical approach. They identified 106 patients over a 15-year period who were undergoing open nephroureterectomy compared with 28 patients who were undergoing a laparoscopic approach during the same period. A total of $20 \%$ of the open group and $14 \%$ of the laparoscopic group were found to have positive nodes. There was no statistical difference in number of nodes removed, number of positive lymph nodes, and lymph node density, dissecting all of the nodal tissue along the ipsilateral great vessel from the crus of the diaphragm to the common iliac lymph nodes. Interaortocaval nodes were removed at the discretion of the surgeon. The median lymph node count was six for the laparoscopic approach and three for the open approach, suggesting that an adequate lymph node dissection can be performed laparoscopically.

Recommendations. Lymphadenectomy can be performed successfully using a laparoscopic approach with outcomes similar to those achieved with the open surgical nephroureterectomy and lymphadenectomy. The current data would suggest that lymph node dissection with nephroureterectomy is a staging procedure, especially in locally advanced disease, useful in guiding the need for adjuvant chemotherapy. There is a suggestion that lymphadenectomy can be therapeutic in $T_{2}$ disease, with increasing numbers of lymph nodes removed and extent of dissection associated with a survival advantage. To better understand the benefits of lymphadenectomy in UTUC, it is essential to standardize the indications for and extent of lymph node dissection performed and evaluate results prospectively. Because of the difficulty with clinical staging of UTUC, at least a limited lymphadenectomy based on tumor location should be performed, with a more extended dissection used for higher stage disease. ${ }^{60}$

\section{Staging}

\section{Renal-cell carcinoma (RCC)}

Current evidence for lymphadenectomy. The role of lymphadenectomy in the management of RCC has fluctuated over time. Most series report that $5 \%$ to $26 \%$ of patients with RCC harbor occult nodal metastases. ${ }^{67}$ Historically, there were limited options for patients with lymph node metastases with only a selected group of patients eligible for high-dose interleukin-2. The development of targeted therapies for RCC has expanded the number of patients who are eligible for adjuvant therapy. Results of the ASSURE study will hopefully provide insight into appropriate selection of patients to treat adjuvantly (clinicaltrials.gov). With this background, it is useful to determine who does and does not need lymphadenectomy and the extent of the lymphadenectomy to be performed. It is likely that lymphadenectomy should be reserved for a selected group of patients, because the morbidity of retroperitoneal dissection is not trivial. ${ }^{68}$

The most useful data come from the European Organization for Research and Treatment of Cancer (EORTC) 30881 randomized phase-III trial that was published recently. Patients who were deemed curable by radical nephrectomy were randomized to radical nephrectomy with or without extended lymphadenectomy. Patients in the nephrectomyonly group underwent lymph node sampling for enlarged lymph nodes only (33 patients, four positive lymph nodes). A total of 732 eligible patients were evaluated, and no significant difference was found in overall survival, time to progression, or progression-free survival between the two groups. ${ }^{69}$ This level I evidence suggests that patients with localized disease do not benefit from extended lymphadenectomy; omitting this decreases the potential morbidity of the procedure and operative time. These findings can be extrapolated to cases amenable to partial nephrectomy without evidence of regional lymphadenopathy.

There are patients that may potentially benefit from the added information provided by a regional lymph node dissection in the absence of metastatic disease. Blute and colleagues ${ }^{70}$ reviewed their experience at the Mayo Clinic in 1652 patients with $\mathrm{pM}_{0}$ disease. Forty-two percent of their patients did not have a lymph node dissection, indicating a possible selection bias. Fewer than $10 \%$ of the cohort had lymph node metastases. In a multivariable model, tumor grade, sarcomatoid differentiation, tumor size $>10 \mathrm{~cm}$, tumor necrosis, and stage were all associated with lymph node metastases. These variables, aside from tumor size and the presence of possible necrosis if noted on imaging, however, are not frequently available preoperatively, making it difficult to use them as guides to decide which patients should undergo lymphadenectomy at the time of nephrectomy. Mass biopsy could provide tumor grade and sarcomatoid differentiation information, but would expose the patient to added morbidity. As such, these data would suggest that patients with tumors $>10 \mathrm{~cm}$ on CT without signs of enlarged lymph nodes or metastatic disease warrant lymphadenectomy.

Joslyn and colleagues ${ }^{67}$ performed a retrospective study using the SEER database. They identified 2831 patients who underwent nephrectomy between 1983 and 1998 and had associated lymphadenectomy information. They reported a decreased overall survival with increasing number of lymph nodes examined. There was no statistical relationship, however, between survival and the number of lymph nodes examined in patients with localized disease. This study suggests that in the absence of lymphadenopathy on CT or at the time of surgery, a lymphadenectomy can be omitted, because the risk of occult lymph node metastases in these patients is $<2 \%$. In patients with regional or distant metastatic disease, this study suggests that the extent of lymphadenectomy does not improve survival. These data are from an era before targeted therapy; currently, it may be important to perform at least a limited lymphadenectomy in the setting of regionally advanced or metastatic disease to improve staging and determine the need for adjuvant therapy. ${ }^{67}$

Feasibility of minimally invasive approaches. Multiple series have addressed the feasibility of lymphadenectomy in the setting of minimally invasive renal surgery. Hemal and associates $^{71}$ reported on a comparison of open vs laparoscopic lymphadenectomy for patients with clinical $\mathrm{T}_{2}$ disease. Of the 41 patients who were undergoing laparoscopic nephrectomy, $73 \%$ had a limited hilar lymphadenectomy that detected only two $(4.9 \%)$ patients with micrometastases. This was com- 
pared with 71 open radical nephrectomies in which $81 \%$ of the patients underwent lymphadenectomy with $9.8 \%$ of the patients identified with micrometastases. As a retrospective evaluation without randomization, selection biases could explain these differences, because patients perceived to have worse disease may have been preferentially selected for an open approach. Despite this, interestingly, there was no difference in 5-year disease-specific or overall survival between the open and laparoscopic cohorts.

Chapman and colleagues ${ }^{68}$ compared laparoscopic nephrectomy with $(n=50)$ or without $(n=50)$ lymphadenectomy in a series of consecutive patients. The lymphadenectomy template was similar to that of a RPLND, with the superior extent being the crus of the diaphragm. In the lymphadenectomy group, a mean of 12.1 lymph nodes were removed. There were no statistically significant differences in complications between the two groups, but there were two cases of chylous ascites in the lymphadenectomy group. A total of $10 \%$ of patients in the lymphadenectomy group were identified with micrometastatic disease. Risk factors included tumors $>7 \mathrm{~cm}, \mathrm{pT}_{3}$ or $\mathrm{pT}_{4}$ disease, and high-grade tumors. The authors demonstrated that an extended lymphadenectomy was feasible using a laparoscopic approach.

Simmons and coworkers ${ }^{72}$ reported on their series of laparoscopic nephrectomies. Of 700 patients, 14 underwent lymphadenectomy at the time of laparoscopic surgery, including 7 patients with known metastatic disease and 1 partial nephrectomy patient. An average of 2.7 lymph nodes were removed in this group, with $57 \%$ of patients identified with node-positive disease. While this node yield is lower than that reported by Chapman and associates, ${ }^{68}$ the authors demonstrate the feasibility of lymphadenectomy in a setting of known advanced disease.

Recommendations. Lymphadenectomy for RCC should be considered a staging procedure without clear therapeutic benefit. The staging information obtained, however, can be useful to predict prognosis and assess the need for adjuvant therapy. Limited or extended laparoscopic lymphadenectomy is feasible but is not routinely performed. It is clear from the EORTC study that patients with localized disease $\left(\leq T_{2}\right)$ on preoperative staging do not benefit from routine lymphadenectomy. Surgeons who use a minimally invasive approach should be facile in lymphadenectomy in the event that intraoperative findings indicate a potential benefit of lymphadenectomy (eg, enlarged perihilar lymph nodes, tumor clearly extending into the perirenal fat).

Based on the limited available literature, lymphadenectomy should be considered if the renal mass is $>7 \mathrm{~cm}$ in greatest dimension, there is evidence of tumor necrosis on CT scan, or if a preoperative biopsy was performed and highgrade RCC is identified. There is no evidence to support an extended lymph node dissection in any setting, and the decision to perform an extended laparoscopic lymphadenectomy should be at the discretion of the surgeon.

\section{Conclusions}

Depending on disease, lymphadenectomy is an important component of the surgical management of urologic malignancies. While the increasing use of minimally invasive techniques for the management of genitourinary cancers has the potential of reduced morbidity, there is an imperative to use lymphadenectomy with these approaches to assess oncologic equivalency to the conventional open surgical approaches. In most circumstances, there is no level 1 evidence to determine the role of lymphadenectomy; however, it is clear that adequate lymphadenectomy is a useful staging tool to guide adjuvant therapy and potentially therapeutic. The literature does demonstrate that lymphadenectomy can be accomplished via minimally invasive approaches with comparable results.

\section{Disclosure Statement}

No competing financial interests exist.

\section{References}

1. Gervasoni JE Jr, Sbayi S, Cady B. Role of lymphadenectomy in surgical treatment of solid tumors: An update on the clinical data. Ann Surg Oncol 2007;14:2443-2462.

2. Farnell MB, Pearson RK, Sarr MG, et al. A prospective randomized trial comparing standard pancreatoduodenectomy with pancreatoduodenectomy with extended lymphadenectomy in resectable pancreatic head adenocarcinoma. Surgery 2005;138:618-630.

3. Sasako M, Sano T, Yamamoto S, et al. D2 lymphadenectomy alone or with para-aortic nodal dissection for gastric cancer. N Engl J Med 2008;359:453-462.

4. Hollenbeck BK, Taub DA, Miller DC, et al. The regionalization of radical cystectomy to specific medical centers. J Urol 2005;174:1385-1389.

5. Yap SA, DeLair SM, Tanaka ST, Kurzrock EA. Current perceptions of resident training in laparoscopic nephrectomy. Urology 2009;73:1067-1071.

6. Protzel C, Alcaraz A, Horenblas S, et al. Lymphadenectomy in the surgical management of penile cancer. Eur Urol 2009;55:1075-1088.

7. Kroon BK, Horenblas S, Lont AP, et al. Patients with penile carcinoma benefit from immediate resection of clinically occult lymph node metastases. J Urol 2005;173:816-819.

8. Hegarty PK, Kayes O, Freeman A, et al. A prospective study of 100 cases of penile cancer managed according to European Association of Urology guidelines. BJU Int 2006;98: 526-531.

9. Catalona WJ. Modified inguinal lymphadenectomy for carcinoma of the penis with preservation of saphenous veins: Technique and preliminary results. J Urol 1988;140:306-310.

10. Bevan-Thomas R, Slaton JW, Pettaway CA. Contemporary morbidity from lymphadenectomy for penile squamous cell carcinoma: The M.D. Anderson Cancer Center Experience. J Urol 2002;167:1638-1642.

11. Bishoff JT, Basler JW, Teichman JM. Endoscopic subcutaneous modified inguinal lymph node dissection (ESMIL) for squamous cell carcinoma of the penis [abstract 301]. J Urol 2003.

12. Tobias-Machado M, Tavares A, Ornellas AA, et al. Video endoscopic inguinal lymphadenectomy: A new minimally invasive procedure for radical management of inguinal nodes in patients with penile squamous cell carcinoma. I Urol 2007;177:953-958.

13. Assimos DG, Jarow JP. Role of laparoscopic pelvic lymph node dissection in the management of patients with penile cancer and inguinal adenopathy. J Endourol 1994;8:365-369.

14. Sotelo R, Sanchez-Salas R, Carmona O, et al. Endoscopic lymphadenectomy for penile carcinoma. J Endourol 2007;21: 364-367. 
15. Yu HY, Madison RA, Setodji CM, et al. Quality of surveillance for stage I testis cancer in the community. I Clin Oncol 2009;27:4327-4332.

16. Donohue JP, Zachary JM, Maynard BR. Distribution of nodal metastases in nonseminomatous testis cancer. I Urol 1982;128:315-320.

17. Albqami N, Janetschek G. Laparoscopic retroperitoneal lymph-node dissection in the management of clinical stage I and II testicular cancer. J Endourol 2005;19:683-692.

18. van den Belt-Dusebout AW, Nuver J, de Wit R, et al. Longterm risk of cardiovascular disease in 5-year survivors of testicular cancer. J Clin Oncol 2006;24:467-475.

19. Rassweiler JJ, Scheitlin W, Heidenreich A, et al. Laparoscopic retroperitoneal lymph node dissection: Does it still have a role in the management of clinical stage I nonseminomatous testis cancer? A European perspective. Eur Urol 2008;54:1004-1015.

20. Carver BS, Shayegan B, Serio A, et al. Long-term clinical outcome after postchemotherapy retroperitoneal lymph node dissection in men with residual teratoma. J Clin Oncol 2007;25:1033-1037.

21. Davol P, Sumfest J, Rukstalis D. Robotic-assisted laparoscopic retroperitoneal lymph node dissection. Urology 2006;67:199.

22. Hellenthal NJ, Ramirez ML, Evans CP, et al. Trends in pelvic lymphadenectomy at the time of radical cystectomy: 1988 to 2004. J Urol 2009;181:2490-2495.

23. Leissner J, Ghoneim MA, Abol-Enein $\mathrm{H}$, et al. Extended radical lymphadenectomy in patients with urothelial bladder cancer: Results of a prospective multicenter study. I Urol 2004;171:139-144.

24. Stein JP, Cai J, Groshen S, Skinner DG. Risk factors for patients with pelvic lymph node metastases following radical cystectomy with en bloc pelvic lymphadenectomy: Concept of lymph node density. J Urol 2003;170:35-41.

25. Herr HW, Bochner BH, Dalbagni G, et al. Impact of the number of lymph nodes retrieved on outcome in patients with muscle invasive bladder cancer. J Urol 2002;167:12951298.

26. Konety BR, Joslyn SA, O'Donnell MA. Extent of pelvic lymphadenectomy and its impact on outcome in patients diagnosed with bladder cancer: Analysis of data from the Surveillance, Epidemiology and End Results Program data base. J Urol 2003;169:946-950.

27. Leissner J, Hohenfellner R, Thuroff JW, Wolf HK. Lymphadenectomy in patients with transitional cell carcinoma of the urinary bladder; significance for staging and prognosis. BJU Int 2000;85:817-823.

28. Poulsen AL, Horn T, Steven K. Radical cystectomy: Extending the limits of pelvic lymph node dissection improves survival for patients with bladder cancer confined to the bladder wall. I Urol 1998;160:2015-2020.

29. Stein JP, Lieskovsky G, Cote R, et al. Radical cystectomy in the treatment of invasive bladder cancer: Long-term results in 1,054 patients. I Clin Oncol 2001;19:666-675.

30. Pruthi RS, Smith A, Wallen EM. Evaluating the learning curve for robot-assisted laparoscopic radical cystectomy. J Endourol 2008;22:2469-2474.

31. Finelli A, Gill IS, Desai MM, et al. Laparoscopic extended pelvic lymphadenectomy for bladder cancer: Technique and initial outcomes. J Urol 2004;172:1809-1812.

32. Haber GP, Gill IS. Laparoscopic radical cystectomy for cancer: Oncological outcomes at up to 5 years. BJU Int 2007;100:137-142.
33. Abraham JB, Young JL, Box GN, et al. Comparative analysis of laparoscopic and robot-assisted radical cystectomy with ileal conduit urinary diversion. I Endourol 2007;21:14731480.

34. Pruthi RS, Wallen EM. Is robotic radical cystectomy an appropriate treatment for bladder cancer? Short-term oncologic and clinical follow-up in 50 consecutive patients. Urology 2008;72:617-622.

35. Wang GJ, Barocas DA, Raman JD, et al. Robotic vs open radical cystectomy: Prospective comparison of perioperative outcomes and pathological measures of early oncological efficacy. BJU Int 2008;101:89-93.

36. Partin AW, Mangold LA, Lamm DM, et al. Contemporary update of prostate cancer staging nomograms (Partin Tables) for the new millennium. Urology 2001;58:843-848.

37. Cerny JC, Farah R, Rian R, Weckstein ML. An evaluation of lymphangiography in staging carcinoma of the prostate. I Urol 1975;113:367-370.

38. Raghavaiah NV, Jordan WP Jr. Prostatic lymphography. J Urol 1979;121:178-181.

39. Gil-Vernet JM. Prostate cancer: Anatomical and surgical considerations. Br J Urol 1996;78:161-168.

40. Bader P, Burkhard FC, Markwalder R. et al. Is a limited lymph node dissection an adequate staging procedure for prostate cancer? J Urol 2002;168:514-518.

41. Heidenreich A, Varga Z, Von Knobloch R. Extended pelvic lymphadenectomy in patients undergoing radical prostatectomy: High incidence of lymph node metastasis. J Urol 2002;167:1681-1686.

42. Joslyn SA, Konety BR. Impact of extent of lymphadenectomy on survival after radical prostatectomy for prostate cancer. Urology 2006;68:121-125.

43. Miyake H, Kurahashi T, Hara I, et al. Significance of micrometastases in pelvic lymph nodes detected by real-time reverse transcriptase polymerase chain reaction in patients with clinically localized prostate cancer undergoing radical prostatectomy after neoadjuvant hormonal therapy. BJU Int 2007;99:315-320.

44. Schumacher MC, Burkhard FC, Thalmann GN, et al. Good outcome for patients with few lymph node metastases after radical retropubic prostatectomy. Eur Urol 2008;54: 344-352.

45. DiMarco DS, Zincke H, Sebo TJ, et al. The extent of lymphadenectomy for pTXNO prostate cancer does not affect prostate cancer outcome in the prostate specific antigen era. J Urol 2005;173:1121-1125.

46. Berglund RK, Sadetsky N, DuChane J, et al. Limited pelvic lymph node dissection at the time of radical prostatectomy does not affect 5-year failure rates for low, intermediate and high risk prostate cancer: Results from CaPSURE. J Urol 2007;177:526-530.

47. Ficarra V, Novara G, Artibani W, et al. Retropubic, laparoscopic, and robot-assisted radical prostatectomy: A systematic review and cumulative analysis of comparative studies. Eur Urol 2009;55:1037-1063.

48. Schroeck FR, Sun L, Freedland SJ, et al. Comparison of prostate-specific antigen recurrence-free survival in a contemporary cohort of patients undergoing either radical retropubic or robot-assisted laparoscopic radical prostatectomy. BJU Int 2008;102:28-32.

49. Prasad SM, Keating NL, Wang Q, et al. Variations in surgeon volume and use of pelvic lymph node dissection with open and minimally invasive radical prostatectomy. Urology 2008;72:647-653. 
50. Zorn KC, Katz MH, Bernstein A, et al. Pelvic lymphadenectomy during robot-assisted radical prostatectomy: Assessing nodal yield, perioperative outcomes, and complications. Urology 2009;74:296-302.

51. Wyler SF, Sulser T, Seifert HH, et al. Laparoscopic extended pelvic lymph node dissection for high-risk prostate cancer. Urology 2006;68:883-887.

52. Silberstein JL, Derweesh IH, Kane CJ. Lymph node dissection during robot-assisted radical prostatectomy: Where do we stand? Prostate Cancer Prostatic Dis 2009;12:227232.

53. Messing EM, Manola J, Sarosdy M, et al. Immediate hormonal therapy compared with observation after radical prostatectomy and pelvic lymphadenectomy in men with node-positive prostate cancer. N Engl J Med 1999;341:17811788.

54. Bhatta-Dhar N, Reuther AM, Zippe C, Klein EA. No difference in six-year biochemical failure rates with or without pelvic lymph node dissection during radical prostatectomy in low-risk patients with localized prostate cancer. Urology 2004;63:528-531.

55. Weight CJ, Reuther AM, Gunn PW, et al. Limited pelvic lymph node dissection does not improve biochemical relapse-free survival at 10 years after radical prostatectomy in patients with low-risk prostate cancer. Urology 2008;71:141145.

56. Roscigno M, Shariat SF, Margulis V, et al. Impact of lymph node dissection on cancer specific survival in patients with upper tract urothelial carcinoma treated with radical nephroureterectomy. I Urol 2009;181:2482-2489.

57. Roscigno M, Shariat SF, Margulis V, et al. The extent of lymphadenectomy seems to be associated with better survival in patients with nonmetastatic upper-tract urothelial carcinoma: How many lymph nodes should be removed? Eur Urol 2009;56:512-518.

58. Bolenz C, Shariat SF, Fernández MI, et al. Risk stratification of patients with nodal involvement in upper tract urothelial carcinoma: Value of lymph-node density. BJU Int 2009;103: 302-306.

59. Kondo $\mathrm{T}$, Nakazawa $\mathrm{H}$, Ito $\mathrm{F}$, et al. Impact of the extent of regional lymphadenectomy on the survival of patients with urothelial carcinoma of the upper urinary tract. I Urol 2007; 178:1212-1217.

60. Miyake H, Hara I, Gohji K, et al. The significance of lymphadenectomy in transitional cell carcinoma of the upper urinary tract. Br J Urol 1998;82:494-498.

61. Capitanio U, Shariat SF, Isbarn H, et al. Comparison of oncologic outcomes for open and laparoscopic nephroureterectomy: A multi-institutional analysis of 1249 cases. Eur Urol 2009;56:1-9.

62. Waldert M, Remzi M, Klingler HC, et al. The oncological results of laparoscopic nephroureterectomy for upper urinary tract transitional cell cancer are equal to those of open nephroureterectomy. BJU Int 2009;103:66-70.

63. Roupret M, Hupertan V, Sanderson KM, et al. Oncologic control after open or laparoscopic nephroureterectomy for upper urinary tract transitional cell carcinoma: A single center experience. Urology 2007;69:656-661.

64. Hsueh TY, Huang YH, Chiu AW, et al. Survival analysis in patients with upper urinary tract transitional cell carcinoma: A comparison between open and hand-assisted laparoscopic nephroureterectomy. BJU Int 2007;99:632-636.

65. Manabe D, Saika T, Ebara S, et al. Comparative study of oncologic outcome of laparoscopic nephroureterectomy and standard nephroureterectomy for upper urinary tract transitional cell carcinoma. Urology 2007;69:457-461.

66. Busby JE, Brown GA, Matin SF. Comparing lymphadenectomy during radical nephroureterectomy: Open versus laparoscopic. Urology 2008;71:413-416.

67. Joslyn SA, Sirintrapun SJ, Konety BR. Impact of lymphadenectomy and nodal burden in renal cell carcinoma: Retrospective analysis of the National Surveillance, Epidemiology, and End Results database. Urology 2005;65:675680.

68. Chapman TN, Sharma S, Zhang S, et al. Laparoscopic lymph node dissection in clinically node-negative patients undergoing laparoscopic nephrectomy for renal carcinoma. Urology 2008;71:287-291.

69. Blom JH, van Poppel H, Maréchal JM, et al. Radical nephrectomy with and without lymph-node dissection: Final Results of European Organization for Research and Treatment of Cancer (EORTC) randomized phase 3 trial 30881. Eur Urol 2009;55:28-34.

70. Blute ML, Leibovich BC, Cheville JC, et al. A protocol for performing extended lymph node dissection using primary tumor pathological features for patients treated with radical nephrectomy for clear cell renal cell carcinoma. J Urol 2004; 172:465-469.

71. Hemal AK, Kumar A, Kumar R, et al. Laparoscopic versus open radical nephrectomy for large renal tumors: A longterm prospective comparison. J Urol 2007;177:862-866.

72. Simmons MN, Kaouk J, Gill IS, Fergany A. Laparoscopic radical nephrectomy with hilar lymph node dissection in patients with advanced renal cell carcinoma. Urology 2007; 70:43-46.

73. Josephson DY, Jacobsohn KM, Link BA, Wilson TG. Roboticassisted endoscopic inguinal lymphadenectomy. Urology 2009;73:167-171.

74. Bhayani SB, Ong A, Oh WK, et al. Laparoscopic retroperitoneal lymph node dissection for clinical stage I nonseminomatous germ cell testicular cancer: A long-term update. Urology 2003;62:324-327.

75. Castillo OA, Urena RD, Pinto IF. Laparoscopic retroperitoneal lymph node dissection for stage I and II NSGCT: 10 years' experience. J Urol 2004;171(suppl):247.

76. Abdel-Aziz KF, Anderson JK, Svatek R, et al. Laparoscopic and open retroperitoneal lymph-node dissection for clinical stage I nonseminomatous germ-cell testis tumors. J Endourol 2006;20:627-631.

77. Neyer M, Peschel R, Akkad T, et al. Long-term results of laparoscopic retroperitoneal lymph-node dissection for clinical stage I nonseminomatous germ-cell testicular cancer. I Endourol 2007;21:180-183.

78. Nielsen ME, Lima G, Schaeffer EM, et al. Oncologic efficacy of laparoscopic RPLND in treatment of clinical stage I nonseminomatous germ cell testicular cancer. Urology 2007;70:1168-1172.

79. Cresswell J, Scheitlin W, Gozen A, et al. Laparoscopic retroperitoneal lymph node dissection combined with adjuvant chemotherapy for pathological stage II disease in nonseminomatous germ cell tumours: A 15-year experience. BJU Int 2008;102:844-848.

80. Skolarus TA, Bhayani SB, Chiang HC, et al. Laparoscopic retroperitoneal lymph node dissection for low-stage testicular cancer. I Endourol 2008;22:1485-1489.

81. Steiner H, Zangerl F, Stöhr B, et al. Results of bilateral nerve sparing laparoscopic retroperitoneal lymph node dissection for testicular cancer. I Urol 2008;180:1348-1353. 
82. Steiner H, Peschel R, Janetschek G, et al. Long-term results of laparoscopic retroperitoneal lymph node dissection: A single-center 10-year experience. Urology 2004;63:550-555.

83. Steiner H, Müller T, Gozzi C, et al. Two cycles of cisplatinbased chemotherapy for low-volume retroperitoneal stage II nonseminomatous germ cell tumours. BJU Int 2006;98:349352.

84. Calestroupat JP, Sanchez-Salas R, Cathelineau X, et al. Postchemotherapy laparoscopic retroperitoneal lymph node dissection in nonseminomatous germ-cell tumor. J Endourol 2009;23:645-650.

85. Permpongkosol S, Lima GC, Warlick CA, et al. Postchemotherapy laparoscopic retroperitoneal lymph node dissection: Evaluation of complications. Urology 2007;69: 361-365.

86. Maldonado-Valadez R, Schilling D, Anastasiadis AG, et al. Post-chemotherapy laparoscopic retroperitoneal lymphnode dissection in testis cancer patients. J Endourol 2007;21: 1501-1504.

87. Stephenson AJ, Gill IS. Laparoscopic radical cystectomy for muscle-invasive bladder cancer: Pathological and oncological outcomes. BJU Int 2008;102:1296-1301.

88. Guru KA, Kim HL, Piacente PM, Mohler JL. Robot-assisted radical cystectomy and pelvic lymph node dissection: Initial experience at Roswell Park Cancer Institute. Urology 2007;69:469-474.
89. Woods M, Thomas R, Davis R, et al. Robot-assisted extended pelvic lymphadenectomy. J Endourol 2008;22:12971302.

Address correspondence to: Alon Z. Weizer, M.D., M.S.

7312 CCC

1500 East Medical Center Drive Ann Arbor, MI 48109

E-mail: aweizer@umich.edu

\begin{aligned} & \multicolumn{1}{c|}{ Abbreviations Used } \\ & $\mathrm{BEP}=$ bleomycin, etoposide, platinum \\ & $\mathrm{CT}=$ computed tomography \\ & $\mathrm{HR}=$ hazard ratio \\ & $\mathrm{ILND}=$ inguinal lymph node dissection \\ & $\mathrm{NCCN}=$ National Comprehensive Cancer Network \\ & $\mathrm{PLND}=$ pelvic lymph node dissection \\ & $\mathrm{PSA}=$ prostate-specific antigen \\ & $\mathrm{RPLND}=$ retroperitoneal lymph node dissection \\ & $\mathrm{RCC}=$ renal-cell carcinoma \\ & $\mathrm{SEER}=$ Surveillance, Epidemiology, and End Results \\ & $\mathrm{UTUC}=$ upper-tract urothelial carcinoma \\ & $\mathrm{VEIL}=$ video endoscopic inguinal lymphadenectomy \end{aligned}

